\title{
Cytogenetic Instability in Childhood Acute Lymphoblastic Leukemia Survivors
}

\author{
María Sol Brassesco, ${ }^{1,2}$ Danilo Jordão Xavier, ${ }^{2}$ Marjori Leiva Camparoto, ${ }^{2}$ \\ Ana Paula Montaldi, ${ }^{2}$ Paulo Roberto D'Auria Vieira de Godoy, ${ }^{2}$ \\ Carlos Alberto Scrideli, ${ }^{1}$ Luiz Gonzaga Tone, ${ }^{1}$ and Elza Tiemi Sakamoto-Hojo ${ }^{2,3}$ \\ ${ }^{1}$ Departamento de Puericultura e Pediatria, Faculdade de Medicina de Ribeirão Preto, Universidade de São Paulo, \\ 14040-901 São Paulo, Brazil \\ ${ }^{2}$ Departamento de Genética, Faculdade de Medicina de Ribeirão Preto, Universidade de São Paulo, 14040-901 São Paulo, Brazil \\ ${ }^{3}$ Departamento de Biologia, Faculdade de Filosofia Ciências e Letras de Ribeirão Preto (FFCLRP-USP), Universidade de São Paulo, \\ Avenida Bandeirantes 3900, 14040-901 Ribeirão Preto, SP, Brazil
}

Correspondence should be addressed to María Sol Brassesco, solbrassesco@hotmail.com and Elza Tiemi Sakamoto-Hojo, etshojo@usp.br

Received 2 June 2010; Accepted 11 August 2010

Academic Editor: Hans Konrad Muller

Copyright (C) 2011 María Sol Brassesco et al. This is an open access article distributed under the Creative Commons Attribution License, which permits unrestricted use, distribution, and reproduction in any medium, provided the original work is properly cited.

\begin{abstract}
Contemporary anticancer therapies have largely improved the outcome for children with cancer, especially for Acute Lymphoblastic Leukemia (ALL). Actually, between $78 \%$ and $85 \%$ of patients achieve complete remission and are alive after 5 years of therapy completion. However, as cure rates increase, new concerns about the late effects of genotoxic treatment emerge, being the risk of developing secondary neoplasias, the most serious life-threatening rising problem. In the present paper, we describe and review the cytogenetic findings in peripheral lymphocytes from ALL survivors, and discuss aspects associated to the occurrence of increased chromosome rearrangements in this growing cohort.
\end{abstract}

\section{Introduction}

Acute lymphoblastic leukemia (ALL) is the most common malignancy in childhood and is associated with excellent outcomes [1]. Currently, $80 \%$ of children with ALL treated in modern centers are alive and disease-free at 5 years [2]. The major contributors to this long-term survival are the improvements in anticancer therapies. However, they have implied the exposure of patients to combinations of chemotherapy and, in some instances, preventive cranial irradiation, which are well-recognized DNA-damaging agents $[3,4]$. Although most ALL survivors are at a lower risk of developing a late effect of therapy when compared with survivors of other pediatric cancers, these children are almost four times more likely than their siblings to develop a severe or life-threatening chronic medical condition [5]. Recently, the number of patients with second malignancies has increased among long-term survivors of pediatric ALL
[6-8]. There is compelling evidence that specific exposure to radiation and chemotherapy are etiologic agents of secondary neoplasia [9].

Chromosomal aberrations, sister chromatid exchanges and micronuclei, which can be detected by cytogenetic analysis, have been used as important biomarkers of genotoxic exposure; moreover, the relevance of increased frequency of chromosome alterations as indicator of malignancy risk is supported by epidemiological studies suggesting that a high frequency of chromosomal aberrations is predictive of an increased likelihood of cancer [10,11]. The genotoxicity of anticancer treatment has been evaluated by somatic cell mutation assays, immediately or shortly after completion of therapy. However, second malignancies in children usually occur several years after treatment [12]. Thus, the cytogenetic analysis in peripheral lymphocytes from cancer survivors, who have been exposed to chemotherapy, provides an opportunity to investigate the in vivo induction and 
persistence of genomic instability in humans, as well as to evaluate the long-term effects of cancer therapy.

In general, hematological malignancies are characterized by recurrent chromosomal aberrations that lead to the formation of gene fusions and the subsequent expression of chimeric proteins with unique properties [13]. Various gene fusions thought to be solely associated with leukemias and lymphomas, such as $\mathrm{t}(12 ; 21)$ ETV6/RUNX1, $\mathrm{t}(9 ; 22)$ $B C R / A B L, \mathrm{t}(14 ; 18) I G H / B C L 2, \mathrm{t}(2 ; 5) N P M-A L K$, and $M L L$ duplications have been detected in normal individuals with negative history of hematologic disorders $[14,15]$. These and other findings suggested that the measurement of gene fusions in peripheral blood lymphocytes within a study group may be used as a sensitive assay for the detection of genomic instability, and may contribute to risk estimation for the development of lymphoid malignancies [16, 17].

During the last years, our group has investigated the existence of chromosome instability in peripheral lymphocytes from ALL survivors by means of fluorescent in situ hybridization (FISH) and molecular biology using leukemia/lymphoma associated gene fusions as putative markers. The proband group comprised 49 individuals aged 5 to 22 years (average $=12$ ), diagnosed and previously treated for childhood ALL at the University Hospital (Faculty of Medicine, Ribeirão Preto, University of São Paulo, Brazil) with combined modality treatment according to the Brazilian Group of Pediatric Leukemia Treatment (GBTLI) [18]. In this protocol, children were treated with a polychemotherapeutic regimen that includes vincristine, dexamethasone, daunorrubicin, L-asparaginase, prednisone, methotrexate, cytosine arabinoside, cyclophosphamide, folinic acid, etoposide (VP-16), teniposide (VM-26), and 6 mercaptopurine. In some cases prophylactic cranial and/or neuroaxis irradiation was also included. The median event-free survival was 3.8 years (range 5 months to 16 years) and there were no relapses. The characterization of patients was described in the work by Brassesco et al. [19].

Frequency of chromosomal aberrations were compared to that observed for control nonsmoking healthy young subjects, aged 18 to 22 years (average $=19.9$ ), who were not occupationally exposed, and had no history of prior or concurrent malignancy. The study was approved by the local Ethics Committee (HC-FMRP Ethics committee Proc. 1135/2000).

The results obtained for the different markers are pooled and described in the following subsections.

\subsection{ETV6/RUNX1 Fusions. FISH for t(12;21) ETV6/RUNX1} was carried out using the LSI TEL/AML1 ES probe (Vysis, Abbott Diagnostics, Maidenhead, United Kingdom) according to the manufacturers' instructions: 1000 interphase nuclei were scored for each sample to determine the degree of positivity for the fusion and the interphase signal patterns of different cell populations. Numbers of red-green fusion signals were recorded corresponding to numbers of copies ETV6/RUNX1, while numerous red and green signals corresponding to RUNX1 and ETV6, respectively, were collectively considered as "extra signals." Our study showed that ALL
TABLE 1: Mean frequency of ETV6/RUNX1 translocations and extra signals obtained for ALL survivors, healthy individuals, and patients at the time of initial diagnosis [20].

\begin{tabular}{lccc}
\hline Groups of individuals & $n$ & $\begin{array}{c}\text { Translocations } \\
\%\end{array}$ & $\begin{array}{c}\text { Extra Signals } \\
\%\end{array}$ \\
\hline ALL survivors & 12 & $3.20 \pm 1.19^{*}$ & $0.76 \pm 0.68$ \\
Healthy individuals & 12 & $1.02 \pm 0.40$ & $0.44 \pm 0.43$ \\
ALL patients at diagnosis & 8 & $0.57 \pm 2.17$ & $0.53 \pm 1.71$ \\
\hline
\end{tabular}

* Statistically different, $P<.05$.

TABLE 2: Translocation frequency for chromosome 12 obtained for controls and childhood ALL survivors. Peripheral blood lymphocyte samples were collected from the patients at variable periods after therapy conclusion.

\begin{tabular}{lcc}
\hline Groups of individuals & $n$ & Translocations \% (mean \pm SD) \\
\hline ALL survivors & 16 & $3.58 \pm 2.77^{*}$ \\
Healthy individuals & 13 & $1.57 \pm 1.65$ \\
\hline
\end{tabular}

* Statistically different, $P<.05$.

survivors $(n=12)$ presented significantly higher frequency of ETV6/RUNX1 fusions (mean $\pm \mathrm{SD}=3.2 \pm 1.19$ ) than those obtained for the control group $(n=12)$ (mean \pm $\mathrm{SD}=1.02 \pm 0.40)$. Extra signals were also detected for both groups although frequencies were similar [20]. Gene fusions incidence in lymphocytes from the group of patients collected months or years after completion of therapy were then compared with those obtained retrospectively for fixed bone marrow samples collected at the time of diagnosis. Two out of ten samples studied were positive for the $t(12 ; 21)$ at diagnosis, however, when these samples were excluded from the statistical analysis, it was observed that the frequency of aberrations obtained for treated patients were significantly higher than those in the untreated bone marrow samples (mean $\pm \mathrm{SD}=0.57 \pm 2.17$ ) (Table 1 ). More interesting, the fusion gene frequency in bone marrow samples was similar to that observed for the control samples, suggesting an influence of previous exposure to anticancer drugs [20].

Also, sequential FISH was performed on lymphocytes from ALL survivors, using chromosome specific library probes for chromosome 12. For one thousand metaphases scored, the frequency of translocations varied between zero and 7.6 (mean $\pm \mathrm{SD}=3.58 \pm 2.77$ ) for the group of patients while for the control group, the frequency was between zero and 5.5/1000 metaphases (mean \pm SD $=1.57 \pm$ 1.65) (Table 2). The statistical analysis revealed a significant difference $(P=.02)$ between the patients and the control group, in accordance with the previous results using locus specific probes for ETV6/RUNX1, providing further evidence that chemotherapy had induced an increase in genomic instability manifested as de novo chromosome aberrations in peripheral blood lymphocytes sampled over years (most cases) after therapy completion. 
TABLE 3: Mean frequency of $M L L$ translocations and extra signals obtained for ALL survivors and healthy individuals (taken from [19]).

\begin{tabular}{lccc}
\hline Groups of individuals & $n$ & $\begin{array}{c}\text { Translocations } \\
\% \\
(\text { mean } \pm \text { SD })\end{array}$ & $\begin{array}{c}\text { Extra Signals } \\
\% \\
(\text { mean } \pm \text { SD })\end{array}$ \\
\hline $\begin{array}{l}\text { Patients Treated with } \\
\text { topoisomerase II } \\
\text { inhibitors }\end{array}$ & 15 & $0.13 \pm 0.14^{*}$ & $0.28 \pm 0.28$ \\
$\begin{array}{l}\text { Patients Treated without } \\
\text { topoisomerase II }\end{array}$ & 31 & $0.09 \pm 0.117^{*}$ & $0.16 \pm 0.168$ \\
$\begin{array}{l}\text { inhibitors } \\
\text { Healthy individuals }\end{array}$ & 49 & $0.04 \pm 0.065$ & $0.18 \pm 0.198$ \\
\hline
\end{tabular}

* Statistically different, $P<.05$.

For both types of probes the role of other covariates such as age at initial diagnosis, inclusion of prophylactic irradiation, and time passed after therapy conclusion were statistically analyzed, showing that neither of these parameters influenced the incidence of chromosomal aberrations.

1.2. MLL Fusions. FISH assessment for the presence of aberrations involving the MLL gene (11q23) was performed using the commercially available probes LSI MLL Break Apart Rearrangement according to the protocol of the manufacturer (Vysis). The expected signal pattern for a normal cell nucleus is two green(yellow)orange signals. In cells harboring $M L L$ translocations, the green and orange signals appear separated without the yellow intersection allowing detection of any translocation at 11q23 irrespectively of the partner involved. One thousand metaphases from 49 ALL long-term survivors and the same number from control individuals were analyzed. Since $M L L$ aberrations are characteristic of secondary leukemias associated with therapy with topoisomerase II inhibitors [21], the group of patients was divided into two subgroups depending on the inclusion or omission of VP-16 and/or VM-26 in the treatment protocol. Our results showed significant $(P=$ .007) differences between the frequency of translocations observed for the groups of patients and controls. These differences were also significant $(P=.006)$ when the groups of patients (independently of the inclusion of VP-16 and/or VM-26) and controls were compared, indicating that higher frequency in the patients cohort might be associated with the therapy with antitumoral drugs, independently of the inclusion of topoisomerase II inhibitors. Extra signals were also scored, however, their frequency did not differ between groups of patients and controls (Table 3) [19].

In parallel, $M L L$ translocations were analyzed by InversePCR and identified by subsequent cloning and sequencing. This methodology is specifically applicable for the identification of rearrangements with known $5^{\prime}$ sequence, but whose $3^{\prime}$ end could be represented by a myriad of translocation partners as is the case of $M L L$ fusions. Basically, DNA is digested by proper restriction enzymes, circularized and then amplified with divergent primers, detecting any segment flanking a known DNA sequence [22]. Individual PCR products were cut, extracted from gels, and subsequently cloned into the pGEM-T vector, further sequencing of PCR products was achieved in order to confirm the veracity of the rearrangements. Eighty-one percent of patients (35 out of 43) presented putative translocations; from those, $91 \%$ corresponded with $\mathrm{t}(4 ; 11)(\mathrm{q} 21 ; \mathrm{q} 23)$, while the other $9 \%$ corresponded with $\mathrm{t}(\mathrm{X} ; 11), \mathrm{t}(8 ; 11)(\mathrm{q} 23 ; \mathrm{q} 23)$, and $\mathrm{t}(11 ; 16)$. The control group was also analyzed by this technique ( $n=100)$, showing putative $M L L$ rearrangements in $49 \%$ of individuals. The presence of two or three putative translocations was statistically higher in the group of patients $(P<.05)$ when compared with the control group. The results were also analyzed regarding the time in complete clinical remission, which did not influence the results. Similarly, the analysis considering other variables (sex, risk of relapse, and inclusion of radiotherapy) did not influence the number of translocations between patients [19].

1.3. IGH/CCND1 Fusions. Locus-specific identifier dualcolor directly labeled $I G H$ (spectrum Green)/CCND1 (Spectrum-Orange) (Vysis) probes were used for detecting the $t(11 ; 14)$ (q13;q32) originated by the juxtaposition of the immunoglobulin gene $(I G H)$ at $14 \mathrm{q} 32$ with the CCND1 gene (cyclin D1, BCL1, or PRAD1), localized at 11q13. The expected signal pattern for a nucleus with $\mathrm{t}(11 ; 14)$ is one green (normal $I G H$ ), one red (normal CCND1), and two red(yellow)green signals (1G1R2Y), due to the fact that the break usually occurs in the middle of the probe marked region, resulting in two fragments of each gene, however, these fragments can be lost, or extra signals of each gene can be detected, resulting in different patterns of signals (2G1R1Y, 1G2R1Y; or 3G2R, 2G3R, in the case of an extra signal). At least 1000 interphase nuclei were analyzed per individual. The group of patients in complete remission $(n=14)$ presented frequency of fusions varying between 0.09 and $0.49 / 100$ cells. These values were significantly higher $(P=.0126)$ compared with those observed for the control group $(n=15)$, which varied from 0 to 0.29 fusions/100 cells. The probes also allowed the detection of extra signals for both genes, with frequency varying from 0.09 to 0.69 signals $/ 100$ cells for patients and from 0 to 0.48 signals/100 cells for controls. As seen for IGH/CCND1 fusions, the frequency of extra signals presented by the group of patients in complete remission were significantly higher $(P=.0282)$ than those presented by healthy individuals. When the inclusion of preventive cranial irradiation was considered no differences in IGH/CCND1 fusions frequency and extra signals were observed between of patients in complete remission and the control group.

A retrospective analysis was also carried out with sequential bone marrow samples collected at the moment of diagnosis from nine of the fourteen ALL patients, with the aim of determining whether the fusion IGH/CCND1 was already present before treatment. The fusion appeared with frequency varying from 0 to $0.41 \mathrm{IGH} / C C N D 1$ fusions/100 cells and from 0 to 2.6 extra signals/100 cells. Interestingly, among these samples, only one patient showed high 
TABLE 4: Mean frequency of IGH/CCND1 translocations and extra signals obtained for ALL survivors, healthy individuals, and patients at the time of initial diagnosis.

\begin{tabular}{|c|c|c|c|}
\hline Groups of individuals & $n$ & $\begin{array}{c}\text { Translocations } \\
\% \\
(\text { mean } \pm \mathrm{SD}) \\
\end{array}$ & $\begin{array}{c}\text { Extra Signals } \\
\% \\
(\text { mean } \pm \mathrm{SD})\end{array}$ \\
\hline ALL survivors & 14 & $0.19 \pm 0.10^{*}$ & $0.29 \pm 0.17$ \\
\hline Healthy individuals & 15 & $0.09 \pm 0.06$ & $0.19 \pm 0.15$ \\
\hline ALL patients at diagnosis & 9 & $0.19 \pm 0.18$ & $0.39 \pm 0.77$ \\
\hline
\end{tabular}

* Statistically different, $P<.05$.

TABLE 5: Mean frequency of $B C R / A B L$ translocations and extra signals obtained for ALL survivors, healthy individuals, and patients at the time of initial diagnosis.

\begin{tabular}{lccc}
\hline Groups of individuals & $n$ & $\begin{array}{c}\text { Translocations } \\
\%\end{array}$ & $\begin{array}{c}\text { Extra Signals } \\
\% \\
(\text { mean } \pm \mathrm{SD})\end{array}$ \\
\hline ALL survivors & 8 & $0.99 \pm 0.55$ & $1.19 \pm 0.97$ \\
Healthy individuals & 8 & $0.49 \pm 0.53$ & $0.25 \pm 0.27$ \\
ALL patients at diagnosis & 4 & $1.20 \pm 0.26^{*}$ & $1.80 \pm 6.18$ \\
\hline
\end{tabular}

* Statistically different, $P<.05$.

frequency of extra signals (2.6/100 cells), which might represent rearrangements of the $I G H$ gene with another partner (Table 4).

The analysis of other variables such as risk of relapse and inclusion of radiotherapy did not show differences in the frequency of $I G H / C C N D 1$ translocations between patients.

1.4. BCR/ABL1 Fusions. FISH for $\mathrm{t}(9 ; 22)$ that juxtaposes the $A B L$ gene at $9 \mathrm{q} 34$ and the $B C R$ gene at $22 \mathrm{q} 11.2$ was carried out using the LSI $B C R / A B L$ ES probe (Vysis) according to the manufacturers' instructions. The hybridization pattern for these probes is two orange and two green signals for normal cells $(2 \mathrm{O} 2 \mathrm{G})$. Cells with $\mathrm{t}(9 ; 22)$ involving the M-bcr show one large orange, one smaller orange (ES), and one fused orange/green signal (2O1G1F), while for translocations involving the minor breakpoint $(\mathrm{m}-\mathrm{bcr})$ appear as one orange, one green, and two fusion signals (1O1G2F) are observed. In this case, samples from 8 ALL survivors and the same number of control individuals were analyzed. The cytogenetic analysis of 1000 metaphases showed translocation frequency varying from 0.236 to 1.627 for treated patients $($ mean $\pm S D=0.99 \pm 0.55)$ and between zero and 1.30 for the control group (mean $\pm \mathrm{SD}=0.49 \pm 0.53$ ). Extra signals were also detected with frequency varying from 0.59 to 3.69 and zero to 0.9 for patients and controls, respectively. However, the statistical analysis was unable to detect differences for translocations and extra signals between the studied groups $(P>.05)$. A retrospective analysis with sequential samples obtained at the time of diagnosis was also performed $(n=4)$. Translocation incidence varied from 0.8 to 1.54 , while extra signals varied from 1.10 to 15.56 ; however, this group was not big enough to establish comparisons with the frequency observed the other groups (Table 5).

\section{Discussion}

Since 1970, the number of cancer survivors in developed countries has tripled and is growing by $2 \%$ each year [23]. Cancer treatments have become much more aggressive as new agents and better supportive therapies are in constant development. The long-term consequences of cancer cure may be significant for the patients who often experience physical or physiological late effects secondary to their previous cancer treatment [4]. Many of these effects may not occur or become clinically symptomatic until many years after completion of therapy, though, the development of a second malignant neoplasm can be the most devastating late effect of cancer treatment.

Childhood-cancer survivors present a higher risk of developing secondary malignancies [24] which characterize the leading cause of death among this cohort [25]. The time of occurrence has not been established, but in general between 3 and 12 percent of the patients develop a subsequent cancer within the first 20 years after therapy conclusion. The most widely recognized treatment-related risk factors are alkylating agents and topoisomerase II inhibitors (epipodophyllotoxins and anthracyclines). The magnitude of the risk associated with these factors depends on several variables such as initial diagnosis, age at diagnosis, the administration schedule, concomitant medications, and genetic predisposition among others [26]. Exposure to alkylating agents or topoisomerase inhibitors during therapy has also been associated with higher risk of developing secondary leukemia among ALL cured patients [27].

Treatment of pediatric ALL consists of several doses of anticancer drugs given weekly or daily for 2-3 years [28]. These drugs cause DNA breaks, facilitating the formation of chromosome rearrangements $[4,29]$. Thus, like de novo leukemias, therapy-related hematological disorders are commonly associated with recurrent aberrations [7]. Chromosome(s) 7 and/or 5 monosomies or deletions are typical of alkylating agent-induced AML, while balanced translocations involving chromosome bands $11 \mathrm{q} 23$ and $21 \mathrm{q} 22$ are associated to previous therapy with DNA-topoisomerase II inhibitors [23] which usually lead to the formation of gene fusions involving MLL [21] and RUNX1 [30], respectively. Translocations involving the latter have been identified in therapy-related secondary leukemias [31] with partners such as ETO (Eight Twenty One) and EVI1 (Ecotropic Viral Integration site 1 oncogene) [32]. Other numerical and structural rearrangements have also been described in different secondary therapy-related neoplasms although at lower frequency, including $\mathrm{t}(3 ; 21)[33], \mathrm{t}(9 ; 22) B C R-A B L 1$ [34], $\mathrm{t}(1 ; 21), \mathrm{t}(3 ; 21), \mathrm{t}(14 ; 21), \mathrm{t}(15 ; 21)$, and $\mathrm{t}(17 ; 21)$ [35].

In the present paper, we pooled a screening for structural aberrations (ETV6/RUNX1, MLL fusions, IGH/CCND1, and $B C R / A B L 1)$ in ALL long-term survivors, compared with control individuals, as evaluated by FISH on interphase nuclei using a specific commercial DNA probes. As shown in Figure 1, all the markers studied showed higher frequency when compared to the control group, thus providing evidence that chemotherapy could have induced an increase in genomic instability manifested as de novo chromosome aberrations 


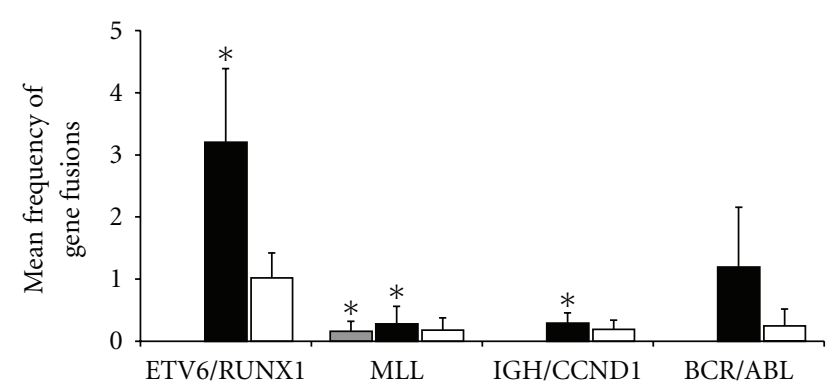

Figure 1: Mean frequency of gene fusions in peripheral lymphocytes from ALL survivors analyzed months or year(s) after therapy completion (black boxes) and healthy donors (white boxes). In the case of $M L L$ fusions the group of survivors was subdivided into patients treated with topoisomerase II inhibitors (grey box) and patients treated without the inclusion of such drugs in the treatment protocol (black box). ${ }^{*} P<.05$. Data for ETV6/RUNX1 and $M L L$ was taken from Brassesco et al. [20] and [19], respectively.

in peripheral blood lymphocytes sampled over years (most cases) after therapy completion. The differences between groups were statistically different for the ETV6/RUNX1, MLL fusions, and IGH/CCND1 markers. In the case of BCR/ABL1, although the rearrangement appeared at higher frequency in ALL survivors, the study groups analyzed were not big enough for the proper comparison.

Mutational studies at the FMS proto-oncogene demonstrated the acquisition of mutation in patients treated with alkylating agents and radiation [3]. The absence of such mutations in biopsies at initial diagnosis suggested that they were somatically acquired after treatment and they appeared with increased frequency in relation to the normal population not exposed to cytotoxic drugs. Similarly, studies of HRPT (hypoxanthine phosphoribosyltransferase) mutations demonstrated significantly elevated frequency in patients treated for pre-B ALL, even two years after therapy completion [12].

Furthermore, rearrangements at 11q23 have been found during treatment of primary cancer, as observed in case of t(11;17) MLL/GASP [36]. Other chromosomal rearrangements such as PML-RARA, CBFB-MYH11, MLL-MLLT1, and $B C R-A B L 1$ have also been observed in Non-Hodgkin Lymphoma patients after high-dose therapy [37] demonstrating the susceptibility of specific genes to mutagenic or carcinogenic agents [38].

Indeed, although translocations might be formed by different mechanisms, several studies have mapped specific chromatin structural elements, such as in vivo topoisomerase II (topo II) cleavage sites, DNAse I hypersensitive sites and scaffold attachment regions (SARs) in the breakpoint regions of relevant genes (including $M L L, A F 4, A F 9, A M L 1$, ETO, CBFB, MYHI1, PML, RARA, TEL, E2A, PBX1, BCR, and $A B L$ ) that are probably associated with preferential breakage after exposure to damage including topoisomerase II inhibitors [39]. In most cases, the breakpoints do not colocalize exactly with topoisomerase II cleavage sites, but map close to or at a variable distance from them. After the induction of DNA cleavage at these sites, the religation of the double strand breaks occurs by the action of complex DNA repair mechanisms, mainly nonhomologous end joining (NHEJ) [39].

Other mechanisms such as illegitimate recombination mediated by $A l u$ repeats and long interspersed nuclear elements (LINEs) have been proposed to participate in the formation of gene fusions $[40,41]$. Illegitimate $V(D) J$ recombination have also been described, with site-specific DNA cleavage at heptamer/nonamer signal sequences with the $I G H$ variable region or the TCR binding region arranged in juxtaposition with other tumor suppressors genes or oncogenes like MYC or CCND1 in lymphoid malignancies $[42,43]$; besides several studies have demonstrated that the exposure to DNA-damaging agents in vitro increase $\mathrm{V}(\mathrm{D}) \mathrm{J}$ rearrangements frequency at cryptic signal sequences other than IGH and TCR loci $[44,45]$.

In addition, the induction of leukemia-associated fusion genes such as $\mathrm{t}(8 ; 21)$ AML1/ETO, $\mathrm{t}(9 ; 22) B C R / A B L$, and t(6;9) DEK/CAM (associated with AML and CML) by high doses of ionizing radiation has been reported in vitro [46] and in patients accidentally exposed to ionizing radiation $[47,48]$.

According to the literature, the inclusion of radiotherapy in different treatment regimens is associated with an increase (2-3 times) in the risk of developing solid tumors [49]. Among the ALL survivors analyzed in the present study, $13(28 \%)$ were submitted to different doses of cranial or neuroaxis $\gamma$-radiation. However, these patients did not show increased translocation frequency (for any marker) compared with patients who were only treated with chemotherapy.

Several authors have demonstrated that the production of unstable aberrations (rings and dicentrics) after radiotherapy decreases with time, and sequential cytogenetic studies performed on peripheral lymphocytes taken from patients treated for different cancers showed a constant decline in chromosomal aberrations [50]. M'kacher et al. [51] demonstrated by chromosome painting that after an initial decrease for up to 6 months after treatment, the frequency of complex rearrangements remained constant for up to 2 years during the follow-up of Hodgkin's lymphoma survivors. In our study, the period after therapy conclusion varied between 5 and 192 months (median event-free survival was 3.8 years), however, the statistical analysis did not show any significant relationship between the frequency of translocations for any of the markers and time passed after therapy conclusion.

In the last years, the treatment directed to the central nervous system has suffered modifications with a tendency of lowering doses and the irradiated area, using dose rationing regimens [52]. In general, protocols have eliminated radiation to the spine, decreased cranial irradiation doses or replaced them with intrathecal and/or systemic drugs such as high-dose methotrexate [5]. Currently, ALL patients at our service are submitted to doses of $18 \mathrm{~Gy}$ (classified as standard risk of relapse) and $24 \mathrm{~Gy}$ (higher risk of relapse), according to the GBTLI protocol [18]. Nonetheless, several epidemiological studies have demonstrated that the risk of t-LMAs or t-MDSs is predominantly associated with chemotherapy and not to radiotherapy, principally emphasizing the relationship 
between specific drugs and chromosome rearrangements [53], as verified for topoisomerase II inhibitors associated to $11 \mathrm{q} 23$ aberrations.

When considering the $M L L$ aberrations, the group of patients presented significantly higher aberration frequency when compared to control individuals, although such differences were independent of the inclusion of VP-16 or VM-26 in the treatment protocol. Some studies have demonstrated that concomitant adjuvant chemotherapy (such as asparaginase, doxorubicin, cyclophosphamide) or antimetabolites (such as mercaptopurine or methotrexate) administration, increase the risk of secondary leukemia [26].

Also, individual predisposing factors, including polymorphisms of detoxification and DNA-repair enzymes that, for instance, reduce the enzymatic activity of thiopurine methyltransferase, a variant of CYP3A that affects production of a DNA-damaging metabolite of epipodophyllotoxin. Other polymorphisms in glutathione S-transferase and $\mathrm{NAD}(\mathrm{P}) \mathrm{H}$ :quinine oxidoreductase (NQO1) are as well associated with increased risk of secondary leukemia after chemotherapy [23].

Since strategies in cancer treatment are continuously evolving, the investigation of rearrangements that could represent a risk factor for the development of secondary neoplasias is important for cancer survivors. Individuals with a history of childhood cancer have 10-20 fold greater risk of developing a secondary malignant neoplasm within the first 20 years after treatment [54] turning therapy-related neoplasias a worldwide problem of major proportions [55, 56].

Between $10 \%$ and $20 \%$ of acute leukemia patients reported in various series have developed a secondary cancer following previous therapy, and considering cured ALL patients, a greater frequency of neoplasias has been observed when compared to normal individuals $[8,57,58]$. None of our patients developed a secondary cancer over 5 years of clinical follow-up. Yet, even though the chromosome aberrations detected in this study appear at low frequency in ALL survivors, there were significant differences when compared to the control group.

Although some studies have demonstrated that some of these fusion genes can lead to a greater cellular proliferation, like $B C R / A B L, E T V 6 / R U N X 1, I G H / B C L-2$, among others, such alterations by themselves are not enough for tumorigenesis, and secondary multiple genetic alterations are necessary [59-61]. Recent studies have also demonstrated the presence of leukemia-related chromosomal translocations in normal individuals, such as $\mathrm{t}(14 ; 18)$ IGH/BCL2 [62-64] and $\mathrm{t}(9 ; 22)[65], \mathrm{t}(12 ; 21)$ ETV6/RUNX1 [14], t(11;14)(p13;q11) LMO2/TCR and $\mathrm{t}(7 ; 14)(\mathrm{q} 34 ; \mathrm{q} 11)$ TCR/TAL2 [42], $\mathrm{t}(15 ; 17)$ $P M L / R A R A$ [66], and MLL partial duplications [15, 67], indicating that the presence of such translocations per se does not define an apparent clinical disease.

Although, it cannot be assumed that the observed therapy-induced genetic damage in the group of ALL survivors is predictive of an increased risk of developing secondary neoplasias, cytogenetic surveillance should be considered to evaluate the occurrence of persistent genome instability in these patients, as well as to associate particular evolving genomic aberrations to the potential risk of secondary malignancies in this cohort.

\section{Acknowledgment}

This work was supported by FAPESP (Grant nos. 01/112253, 02/13317-8, and 03/01915-0), CAPES, and CNPq.

\section{References}

[1] A. Baccichet, S. K. Qualman, and D. Sinnett, "Allelic loss in childhood acute lymphoblastic leukemia," Leukemia Research, vol. 21, no. 9, pp. 817-823, 1997.

[2] C.-H. Pui, L. L. Robison, and A. T. Look, "Acute lymphoblastic leukaemia," The Lancet, vol. 371, no. 9617, pp. 1030-1043, 2008.

[3] A. Baker, P. Cachia, S. Ridge et al., "FMS mutations in patients following cytotoxic therapy for lymphoma," Leukemia Research, vol. 19, no. 5, pp. 309-318, 1995.

[4] J. Byrne, "Long-term genetic and reproductive effects of ionizing radiation and chemotherapeutic agents on cancer patients and their offspring," Teratology, vol. 59, no. 4, pp. 210215, 1999.

[5] P. C. Nathan, K. Wasilewski-Masker, and L. A. Janzen, "Longterm outcomes in survivors of childhood acute lymphoblastic leukemia," Hematology/Oncology Clinics of North America, vol. 23, no. 5, pp. 1065-1082, 2009.

[6] M. A. Smith, L. Rubinstein, and R. S. Ungerleider, "Therapyrelated acute myeloid leukemia following treatment with epipodophyllotoxins: estimating the risks," Medical and Pediatric Oncology, vol. 23, no. 2, pp. 86-98, 1994.

[7] C. A. Felix, "Secondary leukemias induced by topoisomerasetargeted drugs," Biochimica et Biophysica Acta, vol. 1400, no. 1-3, pp. 233-255, 1998.

[8] S. Bhatia, H. N. Sather, O. B. Pabustan, M. E. Trigg, P. S. Gaynon, and L. L. Robison, "Low incidence of second neoplasms among children diagnosed with acute lymphoblastic leukemia after 1983," Blood, vol. 99, no. 12, pp. 4257-4264, 2002.

[9] E. J. Dann and J. M. Rowe, "Biology and therapy of secondary leukaemias," Best Practice and Research, vol. 14, no. 1, pp. 119137, 2001.

[10] S. Bonassi, L. Hagmar, U. Strömberg et al., "Chromosomal aberrations in lymphocytes predict human cancer independently of exposure to carcinogens," Cancer Research, vol. 60, no. 6, pp. 1619-1625, 2000.

[11] H. Norppa, "Cytogenetic biomarkers and genetic polymorphisms," Toxicology Letters, vol. 149, no. 1-3, pp. 309-334, 2004.

[12] S. Koishi, M. Kubota, M. Sawada et al., "Biomarkers in long survivors of pediatric acute lymphoblastic leukemia patients: late effects of cancer chemotherapy," Mutation Research, vol. 422, no. 2, pp. 213-222, 1998.

[13] M. Greaves, "Molecular genetics, natural history and the demise of childhood leukaemia," European Journal of Cancer, vol. 35, no. 2, pp. 173-185, 1999.

[14] M. Eguchi-Ishimae, M. Eguchi, E. Ishii et al., "Breakage and fusion of the TEL (ETV6) gene immature B lymphocytes induced by apoptogenic signals," Blood, vol. 97, no. 3, pp. 737$743,2001$.

[15] J. Bäsecke, F. Griesinger, L. Trümper, and G. Brittinger, "Leukemia- and lymphoma-associated genetic aberrations in healthy individuals," Annals of Hematology, vol. 81, no. 2, pp. 64-75, 2002. 
[16] S. Lipkowitz, V. F. Garry, and I. R. Kirsch, "Interlocus V$\mathrm{J}$ recombination measures genomic instability in agriculture workers at risk for lymphoid malignancies," Proceedings of the National Academy of Sciences of the United States of America, vol. 89, no. 12, pp. 5301-5305, 1992.

[17] I. R. Kirsch and F. Lista, "Lymphocyte-specific genomic instability and risk of lymphoid malignancy," Seminars in Immunology, vol. 9, no. 3, pp. 207-215, 1997.

[18] S. Brandalise, V. Odone, W. Pereira, M. Andrea, M. Zanichelli, and V. Aranega, "Treatment results of three consecutive Brazilian cooperative childhood ALL protocols: GBTLI-80, GBTLI-82 and -85," Leukemia, vol. 7, supplement 2, pp. S142S145, 1993.

[19] M. S. Brassesco, A. P. Montaldi, D. E. Gras et al., "Cytogenetic and molecular analysis of MLL rearrangements in acute lymphoblastic leukaemia survivors," Mutagenesis, vol. 24, no. 2, pp. 153-160, 2009.

[20] M. S. Brassesco, M. L. Camparoto, L. G. Tone, and E. T. Sakamoto-Hojo, "Analysis of ETV6/RUNX1 fusions for evaluating the late effects of cancer therapy in ALL (acute lymphoblastic leukemia) cured patients," Cytogenetic and Genome Research, vol. 104, no. 1-4, pp. 346-351, 2004.

[21] P. D. Aplan, D. S. Chervinsky, M. Stanulla, and W. C. Burhans, "Site-specific DNA cleavage within the MLL breakpoint cluster region induced by topoisomerase II inhibitors," Blood, vol. 87, no. 7, pp. 2649-2658, 1996.

[22] C. J. Betti, M. J. Villalobos, M. O. Diaz, and A. T. M. Vaughan, "Apoptotic triggers initiate translocations within the MLL gene involving the nonhomologous end joining repair system," Cancer Research, vol. 61, no. 11, pp. 4550-4555, 2001.

[23] G. Leone, L. Fianchi, L. Pagano, and M. T. Voso, "Incidence and susceptibility to therapy-related myeloid neoplasms," Chemico-Biological Interactions, vol. 184, no. 1-2, pp. 39-45, 2010.

[24] A. C. MacArthur, J. J. Spinelli, P. C. Rogers, K. J. Goddard, N. Phillips, and M. L. McBride, "Risk of a second malignant neoplasm among 5-year survivors of cancer in childhood and adolescence in British Columbia, Canada," Pediatric Blood and Cancer, vol. 48, no. 4, pp. 453-459, 2007.

[25] S. C. W. Lawless, P. Verma, D. M. Green, and M. C. Mahoney, "Mortality experiences among 15+ year survivors of childhood and adolescent cancers," Pediatric Blood and Cancer, vol. 48, no. 3, pp. 333-338, 2007.

[26] N. Hijiya, K. K. Ness, R. C. Ribeiro, and M. M. Hudson, "Acute leukemia as a secondary malignancy in children and adolescents: current findings and issues," Cancer, vol. 115, no. 1, pp. 23-35, 2009.

[27] L. F. Lopes, B. Camargo, and A. Bianchi, "Late effects of childhood cancer treatment," Revista da Associacao Medica Brasileira, vol. 46, no. 3, pp. 277-284, 2000.

[28] J. G. Blanco, T. Dervieux, M. J. Edick et al., "Molecular emergence of acute myeloid leukemia during treatment for acute lymphoblastic leukemia," Proceedings of the National Academy of Sciences of the United States of America, vol. 98, no. 18, pp. 10338-10343, 2001.

[29] Y.-H. Han, M. J. F. Austin, Y. Pommier, and L. F. Povirk, "Small deletion and insertion mutations induced by the topoisomerase II inhibitor teniposide in $\mathrm{CHO}$ cells and comparison with sites of drug-stimulated DNA cleavage in vitro," Journal of Molecular Biology, vol. 229, no. 1, pp. 52-66, 1993.

[30] M. Stanulla, J. Wang, D. S. Chervinsky, S. Thandla, and P. D. Aplan, "DNA cleavage within the MLL breakpoint cluster region is a specific event which occurs as part of higherorder chromatin fragmentation during the initial stages of apoptosis," Molecular and Cellular Biology, vol. 17, no. 7, pp. 4070-4079, 1997.

[31] J. D. Rowley, "The role of chromosome translocations in leukemogenesis," Seminars in Hematology, vol. 36, no. 4, pp. 59-72, 1999.

[32] M. L. Loh, L. B. Silverman, M. L. Young et al., "Incidence of TEL/AML1 fusion in children with relapsed acute lymphoblastic leukemia," Blood, vol. 92, no. 12, pp. 4792-4797, 1998.

[33] S. W. Hiebert, W. Sun, J. N. Davis et al., "The t(12;21) translocation converts AML-1B from an activator to a repressor of transcription," Molecular and Cellular Biology, vol. 16, no. 4, pp. 1349-1355, 1996.

[34] M. Hattori, M. Tanaka, Y. Yamazaki, Y. Nakahara, K. Tsushita, and M. Utumi, "Detection of major and minor bcr/abl fusion gene transcripts in a patient with acute undifferentiated leukemia secondary to treatment with an alkylating agent," Leukemia Research, vol. 19, no. 6, pp. 389-396, 1995.

[35] D. Roulston, R. Espinosa III, G. Nucifora, R. A. Larson, M. M. Le Beau, and J. D. Rowley, "CBFA2(AML1) translocations with novel partner chromosomes in myeloid leukemias: association with prior therapy," Blood, vol. 92, no. 8, pp. 2879-2885, 1998.

[36] M. D. Megonigal, N.-K. V. Cheung, E. F. Rappaport et al., "Detection of leukemia-associated MLL-GAS7 translocation early during chemotherapy with DNA topoisomerase II inhibitors," Proceedings of the National Academy of Sciences of the United States of America, vol. 97, no. 6, pp. 2814-2819, 2000.

[37] J. Basecke, M. Podleschny, A. Becker et al., "Therapyassociated genetic aberrations in patients treated for nonHodgkin lymphoma," British Journal of Haematology, vol. 141, no. 1, pp. 52-59, 2008.

[38] K. Seeger, H.-P. Adams, D. Buchwald et al., "TEL-AML1 fusion transcript in relapsed childhood acute lymphoblastic leukemia," Blood, vol. 91, no. 5, pp. 1716-1722, 1998.

[39] Y. Zhang and J. D. Rowley, "Chromatin structural elements and chromosomal translocations in leukemia," DNA Repair, vol. 5, no. 9-10, pp. 1282-1297, 2006.

[40] M. P. Strout, G. Marcucci, C. D. Bloomfield, and M. A. Caligiuri, "The partial tandem duplication of ALL1 (MLL) is consistently generated by Alu-mediated homologous recombination in acute myeloid leukemia," Proceedings of the National Academy of Sciences of the United States of America, vol. 95, no. 5, pp. 2390-2395, 1998.

[41] S. P. Whitman, M. P. Strout, M. Guido et al., "The partial nontandem duplication of the MLL (ALL1) gene is a novel rearrangement that generates three distinct fusion transcripts in B-cell acute lymphoblastic leukemia," Cancer Research, vol. 61 , no. 1 , pp. 59-63, 2001.

[42] R. Marculescu, T. Le, P. Simon, U. Jaeger, and B. Nadel, "V(D)J-mediated translocations in lymphoid neoplasms: a functional assessment of genomic instability by cryptic sites," Journal of Experimental Medicine, vol. 195, no. 1, pp. 85-98, 2002.

[43] A. K. Abbas, A. H. Lichtman, and J. S. Pober, Cellular and Molecular Immunology, W. B. Saunders, Philadelphia, Pa, USA, 5th edition, 2003.

[44] C.-L. Chen, J. C. Fuscoe, Q. Liu, and M. V. Relling, "Etoposide causes illegitimate $\mathrm{V}(\mathrm{D}) \mathrm{J}$ recombination in human lymphoid leukemic cells," Blood, vol. 88, no. 6, pp. 2210-2218, 1996.

[45] R. L. Pinsonneault, P. M. Vacek, J. P. O’Neill, and B. A. Finette, "Induction of V(D)J-mediated recombination of an 
extrachromosomal substrate following exposure to DNAdamaging agents," Environmental and Molecular Mutagenesis, vol. 48, no. 6, pp. 440-450, 2007.

[46] M. W. N. Deininger, S. Bose, J. Gora-Tybor, X.-H. Yan, J. M. Goldman, and J. V. Melo, "Selective induction of leukemiaassociated fusion genes by high-dose ionizing radiation," Cancer Research, vol. 58, no. 3, pp. 421-425, 1998.

[47] R. Hromas, R. Shopnick, H. G. Jumean, C. Bowers, M. VarellaGarcia, and K. Richkind, "A novel syndrome of radiationassociated acute myeloid leukemia involving AML1 gene translocations," Blood, vol. 95, no. 12, pp. 4011-4013, 2000.

[48] R. Hromas, T. Busse, A. Carroll et al., "Fusion AML1 transcript in a radiation-associated leukemia results in a truncated inhibitory AML1 protein," Blood, vol. 97, no. 7, pp. 2168-2170, 2001.

[49] K. Kawai and H. Akaza, "Treatment of testicular and second cancer," Gan to Kagaku Ryoho, vol. 26, no. 13, pp. 2021-2028, 1999.

[50] E. J. Tawn, C. A. Whitehouse, and F. A. Martin, "Sequential chromosome aberration analysis following radiotherapyno evidence for enhanced genomic instability," Mutation Research, vol. 465, no. 1-2, pp. 45-51, 2000.

[51] R. M'kacher, T. Girinsky, S. Koscielny et al., "Baseline and treatment-induced chromosomal abnormalities in peripheral blood lymphocytes of Hodgkin's lymphoma patients," International Journal of Radiation Oncology Biology Physics, vol. 57, no. 2, pp. 321-326, 2003.

[52] J. Nachman, H. N. Sather, J. M. Cherlow et al., "Response of children with high-risk acute lymphoblastie leukemia treated with and without cranial irradiation: a report from the Children's Cancer Group," Journal of Clinical Oncology, vol. 16, no. 3, pp. 920-930, 1998.

[53] M. K. Andersen, B. Johansson, S. O. Larsen, and J. PedersenBjergaard, "Chromosomal abnormalities in secondary MDS and AML. Relationship to drugs and radiation with specific emphasis on the balanced rearrangements," Haematologica, vol. 83, no. 6, pp. 483-488, 1998.

[54] L. Löning, M. Zimmermann, A. Reiter et al., "Secondary neoplasms subsequent to Berlin-Frankfurt-Munster therapy of acute lymphoblastic leukemia in childhood: significantly lower risk without cranial radiotherapy," Blood, vol. 95, no. 9, pp. 2770-2775, 2000.

[55] L. L. Robison and S. Bhatia, "Late-effects among survivors of leukaemia and lymphoma during childhood and adolescence," British Journal of Haematology, vol. 122, no. 3, pp. 345-359, 2003.

[56] J. Jazbec, P. Ećimović, and B. Jereb, "Second neoplasms after treatment of childhood cancer in Slovenia," Pediatric Blood and Cancer, vol. 42, no. 7, pp. 574-581, 2004.

[57] S. Bhatia, L. L. Robison, O. Oberlin et al., "Breast cancer and other second neoplasms after childhood Hodgkin's disease," New England Journal of Medicine, vol. 334, no. 12, pp. 745751, 1996.

[58] R. Mody, S. Li, D. C. Dover et al., "Twenty-five-year follow-up among survivors of childhood acute lymphoblastic leukemia: a report from the Childhood Cancer Survivor Study," Blood, vol. 111, no. 12, pp. 5515-5523, 2008.

[59] K. F. Wong, "11q13 is a cytogenetically promiscuous site in hematologic malignancies," Cancer Genetics and Cytogenetics, vol. 113, no. 1, pp. 93-95, 1999.

[60] F. Bernardin, Y. Yang, R. Cleaves et al., "TEL-AML1, expressed from $\mathrm{t}(12 ; 21)$ in human acute lymphocytic leukemia, induces acute leukemia in mice," Cancer Research, vol. 62, no. 14, pp. 3904-3908, 2002.
[61] D. De Jong, "Molecular pathogenesis of follicular lymphoma: a cross talk of genetic and immunologic factors," Journal of Clinical Oncology, vol. 23, no. 26, pp. 6358-6363, 2005.

[62] Y. Liu, A. M. Hernandez, D. Shibata, and G. A. Cortopassi, "BCL2 translocation frequency rises with age in humans," Proceedings of the National Academy of Sciences of the United States of America, vol. 91, no. 19, pp. 8910-8914, 1994.

[63] M. Yasukawa, S. Bando, G. Dölken et al., "Low frequency of BCL-2/JH translocation in peripheral blood lymphocytes of healthy Japanese individuals," Blood, vol. 98, no. 2, pp. 486$488,2001$.

[64] K. E. Summers, L. K. Goff, A. G. Wilson, R. K. Gupta, T. A. Lister, and J. Fitzgibbon, "Frequency of the Bcl-2/IgH rearrangement in normal individuals: implications for the monitoring of disease in patients with follicular lymphoma," Journal of Clinical Oncology, vol. 19, no. 2, pp. 420-424, 2001.

[65] S. Bose, M. Deininger, J. Gora-Tybor, J. M. Goldman, and J. V. Melo, "The presence of typical and atypical BCR-ABL fusion genes in leukocytes of normal individuals: biologic significance and implications for the assessment of minimal residual disease," Blood, vol. 92, no. 9, pp. 3362-3367, 1998.

[66] A. S. Quina, P. Gameiro, M. S. D. Costa, M. Telhada, and L. Parreira, "PML-RARA fusion transcripts in irradiated and normal hematopoietic cells," Genes Chromosomes and Cancer, vol. 29, no. 3, pp. 266-275, 2000.

[67] S. Schnittger, B. Wörmann, W. Hiddemann, and F. Griesinger, "Partial tandem duplications of the MLL gene are detectable in peripheral blood and bone marrow of nearly all healthy donors," Blood, vol. 92, no. 5, pp. 1728-1734, 1998. 

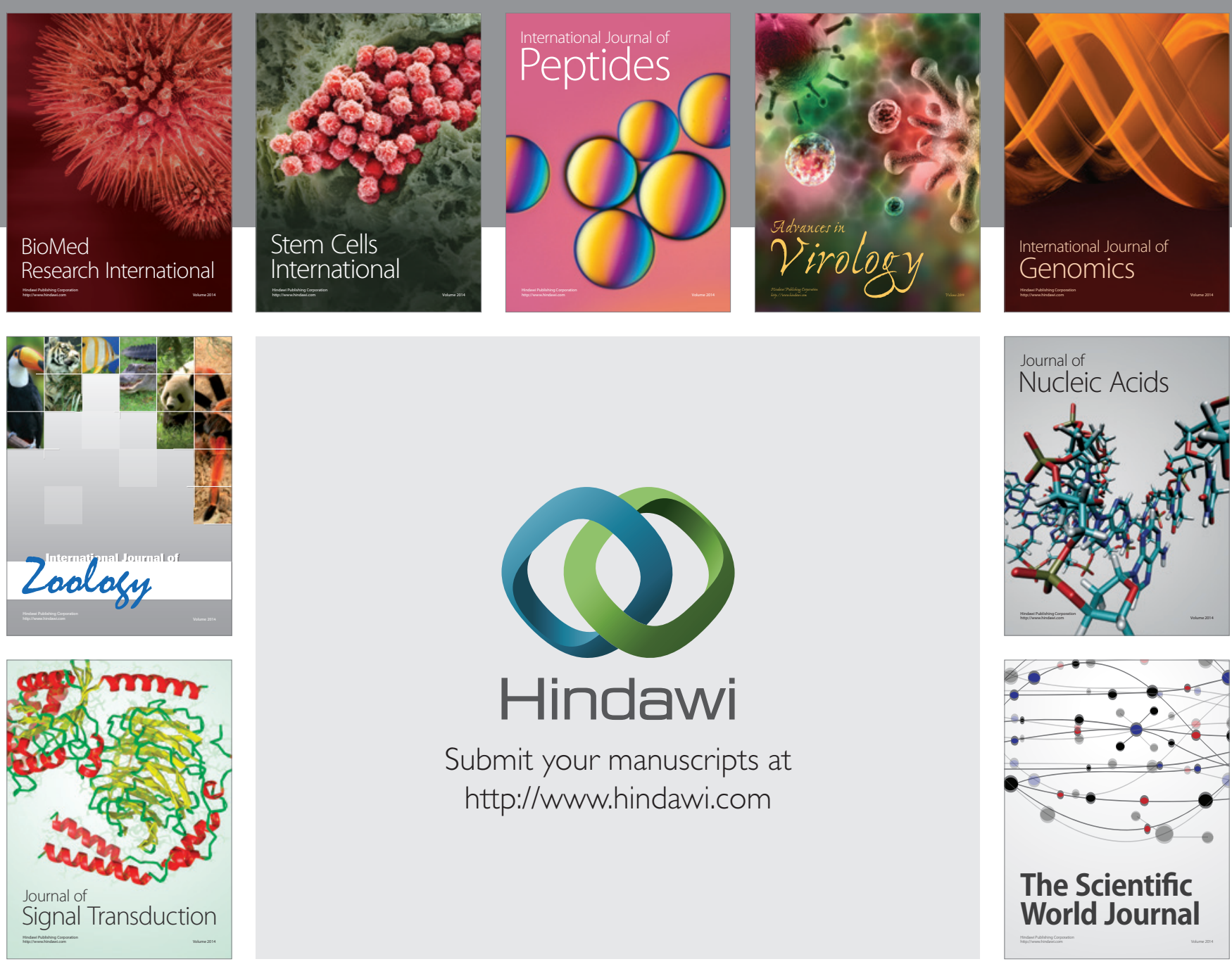

Submit your manuscripts at

http://www.hindawi.com
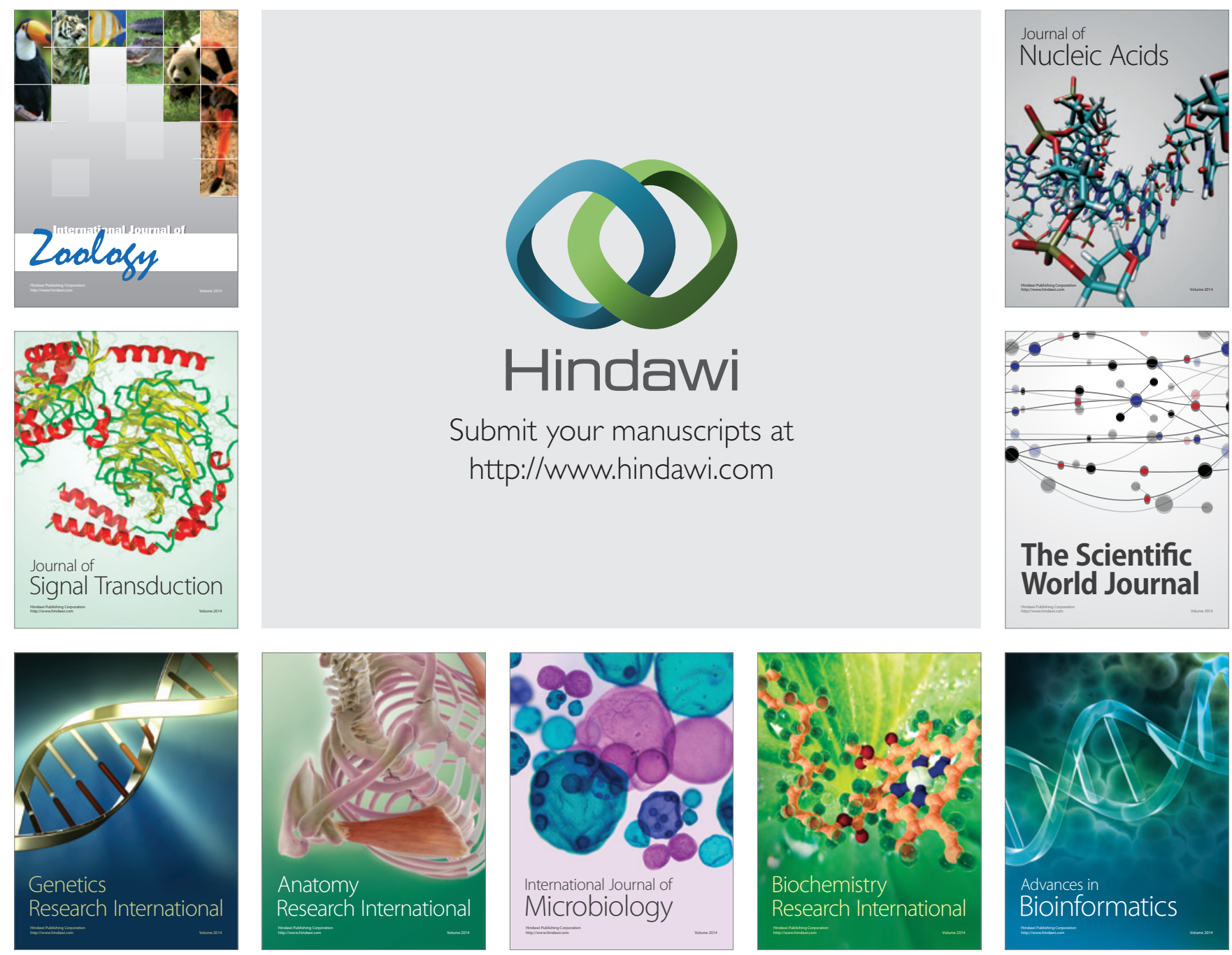

The Scientific World Journal
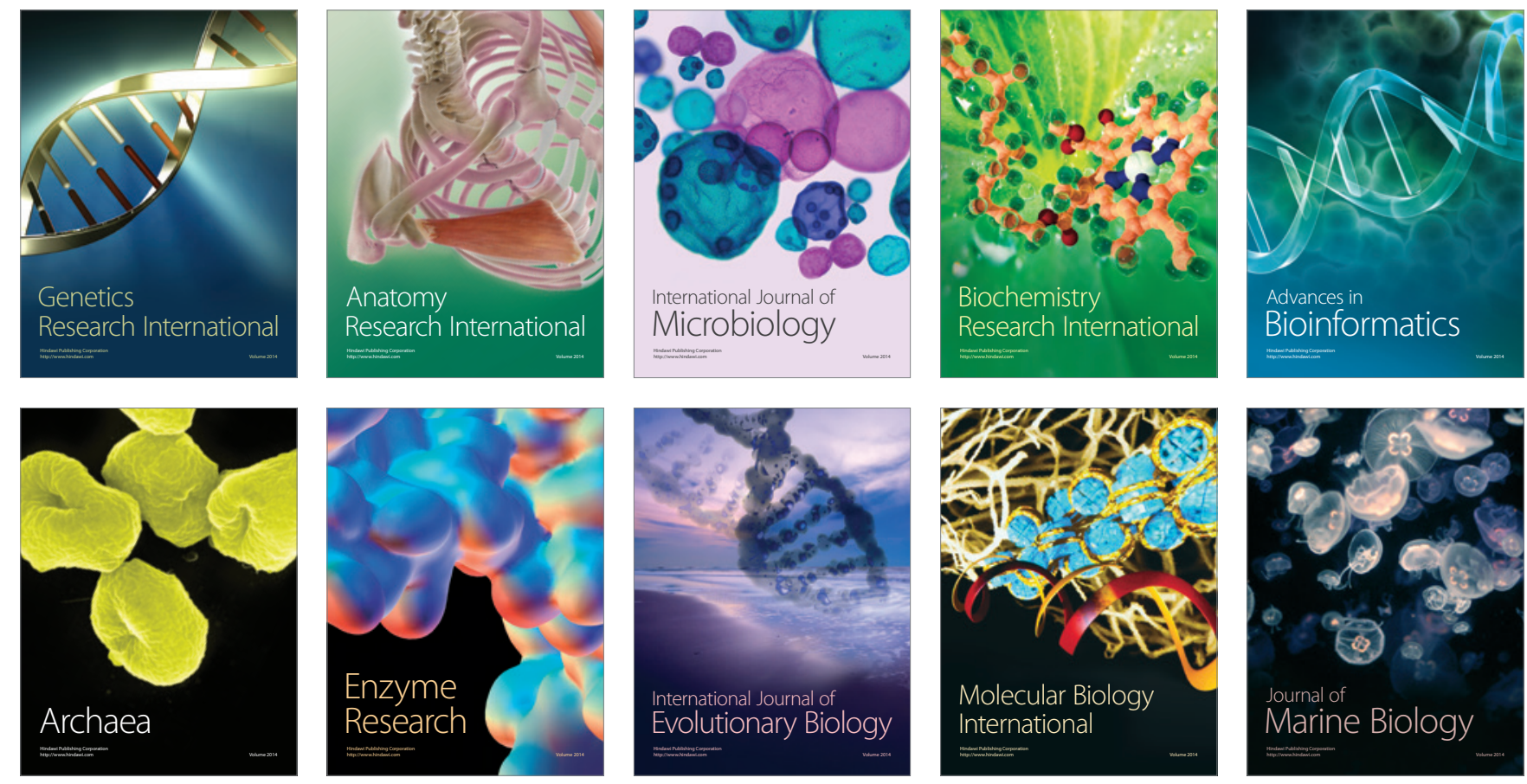